\title{
A Spline-Based Framework for Perfect Reconstruction AM-FM Models
}

\author{
Roy A. Sivley and Joseph P. Havlicek \\ School of Electrical and Computer Engineering, University of Oklahoma, Norman, OK 73019 USA
}

\begin{abstract}
For the first time, we present a multicomponent perfect reconstruction AM-FM image model that is fully consistent with human visual perception. We design a separable perfect reconstruction wavelet filterbank based on the discrete Coiflet and implement it in a maximally decimated parallel structure characterized by excellent joint space-frequency localization. Using the zeros in the channel response magnitudes, we further decompose each channel into a sum of highly localized, non-separable orientation selective subchannels. We fit the responses of these sub-channels with nonlinear spline models and apply error-free continuous demodulation algorithms to obtain a new perfect reconstruction AM-FM model. This is significant because the new model could be used as the basis for a new theory of modulation domain image processing.
\end{abstract}

\section{Introduction}

AM-FM models provide a modulation domain image characterization that has proven useful in a variety of applications including jointly localized analysis, texture segmentation and classification, edge detection and image enhancement, 3-D shape from texture, texture-based stereopsis, fingerprint classification, content-based retrieval, and regeneration of occluded and damaged textures [9]. For a complexvalued image $t(\mathbf{x})$, possibly obtained from a real image using the multidimensional Hilbert transform described in [8], the $K$-component AM-FM image model is given by

$$
t(\mathbf{x})=\sum_{m=1}^{K} y_{m}(\mathbf{x})=\sum_{m=1}^{K} a_{m}(\mathbf{x}) \exp \left[j \varphi_{m}(\mathbf{x})\right] .
$$

Manifest in each AM-FM component $y_{m}(\mathbf{x})$ in (1) is a frequency modulation (FM) function $\nabla \varphi_{m}(\mathbf{x})$ describing the local texture orientation and granularity and an amplitude modulation (AM) function $a_{m}(\mathbf{x})$ describing the local texture contrast. The collection of functions

This work was supported in part by the U.S. Army Research Laboratory and the U.S. Army Research Office under grant W911NF-04-1-0221. $\left\{a_{m}(\mathbf{x}), \nabla \varphi_{m}(\mathbf{x})\right\}_{m \in[1, K]}$ defines the modulation domain image representation, which has been estimated previously using both continuous and discrete algorithms [7,11].

Because of the nonlinearity of the model (1), all previous discrete AM-FM demodulation algorithms have been based on approximations that are generally accurate when applied to image components $y_{m}(\mathbf{x})$ that are locally coherent in the sense of being sufficiently smooth over sufficiently small neighborhoods (a notion made precise in [7, 12, 13]). However, existing discrete algorithms are prone to large approximation errors when the components are not locally coherent. Hence, the success of the whole approach depends on having a filterbank that will decompose the image $t(\mathbf{x})$ into a sum of locally coherent components as indicated in (1). As shown in [1,7], this implies that the filters must possess a high degree of joint localization in space and frequency. Gabor filters have often been used for this reason.

What we are really after is a computational framework capable of supporting a general theory for: 1) representing images in the modulation domain according to (1),2) performing signal processing in the modulation domain, and 3 ) reconstructing the processed images via (1). There are two reasons that existing demodulation algorithms cannot provide this framework. First, a filterbank with good joint localization is needed to obtain locally coherent components that will minimize the approximation errors occurring during demodulation. However, this generally precludes the possibility of the filterbank being a perfect reconstruction (PR) system; hence, PR would not be possible even if there were no demodulation errors. Second, even when Gabor filters - which are the limiting case of maximal joint localization but cannot provide PR - are applied to a practical image, there will generally be a small number of pixels where one or more of the components $y_{m}(\mathbf{x})$ fail to be locally coherent and large-scale demodulation errors result. Sophisticated post processing must be applied on a case by case basis when this occurs, a fact which has severely limited widespread acceptance of the approach.

What is needed is: 1) a PR filterbank (PRF) that yields locally coherent components reflecting the visually significant structure in the image and 2) an error-free demodulation algorithm delivering AM, FM, and PM (phase modula- 
tion) estimates consistent with each other as well as with the pixel values in the original image. The first PR AM-FM image model was given recently in [9]. However, that model failed to provide FM functions in good agreement with human visual perception because all filterbank channels were not sufficiently localized and the demodulation algorithms were inconsistent in a sense we discuss in Section 3.

Here, we present a new PR AM-FM image model exhibiting superior agreement with human visual perception. We implement a separable 2-D Coiflet PRF in a maximally decimated parallel structure having excellent joint localization as described in [18]. Making use of zeros in the response magnitudes, we decompose each channel into a sum of highly localized, non-separable but orientation selective sub-channels. We then apply a new, high-quality 2-D unwrapping algorithm to the phase of each channel. The unwrapped phases are fit with splines which are differentiated analytically to obtain the phase gradient fields. Finally, the AM functions are obtained using the error-free discrete algorithm given in [7].

\section{Perfect Reconstruction Filterbank}

Gabor or similar filters have typically been used in computing multicomponent AM-FM models having demonstrable utility in image analysis applications, but which cannot generally provide the high-quality image reconstructions needed for synthesis applications. Nevertheless, approximate multicomponent AM-FM reconstructions were obtained in $[7,10]$. In this paper, we employ a PRF that is similar to the one described in [19] but which uses a six-point Coiflet mother wavelet because of its superior joint localization. We start with a standard dyadic four-level 1-D wavelet transform. The Noble identities are then used to construct an equivalent maximally decimated parallel system comprising a bank of analysis filters, intermediate down- and up-samplers, and a bank of synthesis filters.

The 1-D analysis and synthesis filterbanks can be applied to the image rows and columns to implement a separable 2D PRF system. However, as illustrated in Figs. 1(a) and 2(a) - which show magnitude frequency responses for two of the separable 2-D channels - there are two reasons that this will not produce a decomposition (1) of the image into locally coherent AM-FM components that capture the visually significant nonstationary structure. First, separable filters are inherently incapable of providing orientation selectivity. Second, an undesirable side effect of the Noble identities is that up-sampling of the analysis and synthesis quadrature mirror filters tends to introduce significant sidelobes. Thus, the components delivered by filters of this type generally fail to be locally coherent and visually meaningful because they contain a wide range of magnitude frequencies and a full spectrum of orientations.
Our solution to this problem is based on the loci of zeros and the symmetry of the lobes that appear in the magnitude responses of Figs. 1(a) and 2(a). In fact, all separable PRF channels of this type exhibit regular patterns of zeros that can be used to partition the lobes into conjugate symmetric pairs. Each such pair constitutes a non-separable subchannel that is orientation selective and highly Gabor-like in its joint localization. Thus, e.g., we decompose each of the separable channels in Figs. 1(a) and 2(a) into 32 nonseparable but orientation selective sub-channels. Two such sub-channels are shown in Fig. 1(b) and 2(b). The locally coherent AM-FM components that result when these two sub-channels are applied to the familiar images of Figs. 1(c) and 2(c) are given in Figs. 1(d) and 2(d), respectively. Implicit in this approach is the fact that the multicomponent model (1) is applied between the analysis and synthesis filterbanks, so that each channel of the analysis bank is interpreted as one component $y_{m}(\mathbf{x})$ which must be processed through the corresponding synthesis channel prior to summing as in (1) to reconstruct the original image.

\section{Consistent Demodulation}

Given a decomposition of the image into suitable components $y_{m}(\mathbf{x})$, the goal is to compute error-free estimates of the modulating functions $a_{m}(\mathbf{x})$ and $\nabla \varphi_{m}(\mathbf{x})$ from which the image can be perfectly reconstructed. In the continuous case where $t: \mathbb{R}^{n} \rightarrow \mathbb{C}$, these functions are given by [7]

$$
\begin{gathered}
a_{m}(\mathbf{x})=\left|y_{m}(\mathbf{x})\right|, \\
\nabla \varphi_{m}(\mathbf{x})=\operatorname{Re}\left[\frac{\nabla y_{m}(\mathbf{x})}{j y_{m}(\mathbf{x})}\right] .
\end{gathered}
$$

The AM algorithm (2) may also be applied directly in the discrete case. However, because the gradient operator in (3) is undefined for discrete images, the FM algorithm must be adapted to the discrete case. Similar to what was done in [4] where discrete signals were fit with spline models so that the continuous Teager-Kaiser energy operator could be applied rigorously to the splines, our approach has been to fit the sampled locally coherent AM-FM components with smooth interpolants for which the derivative and integral are rigorously defined.

The main caveat with this approach is that the phase samples $\varphi_{m}(\mathbf{k})$ are available from the observed component $y_{m}(\mathbf{x})$ only as principal values in the range $(-\pi, \pi]$. This principal phase branch exhibits severe discontinuities that arise solely from branch cuts in the arctan function used to compute it. The FM functions obtained by directly differentiating the principal phase branch will be dominated by high instantaneous frequencies that do not correspond to any visually meaningful structure in the image. While this fact in no way prevents us from obtaining a mathematically valid 
PR AM-FM model, realizing our goal of a meaningful AMFM model that corresponds well to the visually significant structure in the image absolutely requires multidimensional phase unwrapping, which has received considerable attention in the context of SAR interferometry, magnetic resonance imaging, and network flow analysis [2, 5, 6, 15-17]. Nearly all existing phase unwrapping algorithms assume that the inter-pixel phase differences are bounded by $\pi$ radians, and many methods have been proposed to identify and accommodate phase aliased image regions that violate this assumption [6,15-17] or to find the phase that minimizes a given functional norm $[2,5]$. Unfortunately, phase aliasing is quite common for practical images, and, rather than assuming a bound on the phase differences, we prefer to unwrap the phase in a manner that is consistent with visual perception of the modulated image structure.

To guide the phase unwrapping for a given component $y_{m}(\mathbf{x})$, we estimate the nonstationary structure by computing either the phase gradient $\nabla \varphi_{m}(\mathbf{x})$ or the vector of second phase derivatives $\partial^{2} \varphi_{m}(\mathbf{x})$. This is done by applying the tensor product spline framework of Unser et al. [20,21] to the component $y_{m}(\mathbf{k})$ and computing either the gradient in (3) or the second phase derivative vector

$$
\partial^{2} \varphi_{m}(\mathbf{x})=\operatorname{Re}\left[\frac{\partial^{2} y_{m}(\mathbf{x})}{j y_{m}(\mathbf{x})}\right]-2 \nabla \varphi_{m}(\mathbf{x}) \frac{\nabla a_{m}(\mathbf{x})}{a_{m}(\mathbf{x})} .
$$

It is important to note that the differentials defined by (3) and (4) are rigorous for any $\mathrm{C}^{2}$ interpolant, although the resulting estimates $\nabla \widehat{\varphi_{m}}(\mathbf{x})$ and $\partial^{2} \widehat{\varphi_{m}}(\mathbf{x})$ will not generally be the same as those obtained by analytically differentiating an interpolant of the unwrapped phase $\varphi_{m}(\mathbf{k})$. This is due to the use of products and quotients of the interpolants $y_{m}(\mathbf{x}), \nabla y_{m}(\mathbf{x})$, and $\partial^{2} y_{m}(\mathbf{x})$ in (3) and (4) and prevents the phase $\varphi_{m}(\mathbf{x})$ from being directly calculated from $\nabla \widehat{\varphi_{m}}(\mathbf{x})$ or $\partial^{2} \widehat{\varphi_{m}}(\mathbf{x})$ by an integration scheme. Instead, these phase differentials are used to compute the unwrapped phase samples $\psi_{m}(\mathbf{k})$ that minimize the leastsquares error function

$$
\varepsilon=\sum_{i=1}^{2} \sum_{\mathbf{k}}\left(\psi_{m}(\mathbf{k}) * h_{n}\left(\mathbf{e}_{i}^{T} \mathbf{k}\right)-\mathbf{e}_{i}^{T} \partial^{n} \widehat{\varphi_{m}}(\mathbf{k})\right)^{2},
$$

where $\partial^{n} \widehat{\varphi_{m}}(\mathbf{k})$ is the phase differential estimated from (3) or (4), $h_{n}(k)$ is the corresponding 1-D $n^{t h}$-derivative spline filter from [20], and $\mathbf{e}_{i}$ is the unit vector in the $k_{i}$ direction.

Least-squares phase unwrapping has been previously developed using finite phase differences under the assumption that such differences are bounded by $\pi[6,15-17]$; a solution similar to minimizing (5) with the first phase derivative was given in [14]. It can be shown that the DFT of the phase which minimizes (5) is given by

$$
\Psi_{m}(\mathbf{k})=\frac{\sum_{i=1}^{2} \Lambda_{i}(\mathbf{k}) H_{n}^{*}\left(\mathbf{e}_{i}^{T} \mathbf{k}\right)}{\sum_{i=1}^{2}\left|H_{n}\left(\mathbf{e}_{i}^{T} \mathbf{k}\right)\right|^{2}}
$$

where $\Lambda_{i}(\mathbf{k})$ is the DFT of the phase differential estimate $\mathbf{e}_{i}^{T} \partial^{n} \widehat{\varphi_{m}}(\mathbf{k})$, and $H_{n}(k)$ is the frequency response of the $n^{\text {th }}$-derivative spline filter sampled at discrete frequencies. Using the B-splines described in [20], it can also be shown that any first derivative spline filter $H_{1}(z)$ will have zeros at $z= \pm 1$ and that any second derivative spline filter $H_{2}(z)$ will have a zero only at unity. These zeros cause the phase solution in (6) to be ambiguous (i.e., the denominator is zero), and it is an oddity of the discrete derivatives that the phase solution obtained using the second phase differential $\partial^{2} \widehat{\varphi_{m}}(\mathbf{x})$ will be unique up to an additive constant because only the DC coefficient is ambiguous. If the least-squares unwrapping has no residual error (i.e., $\varepsilon=0$ ), this additive constant may be calculated from knowledge of the principal phase values; however, this is seldom the case in practice. In contrast, when using the first phase differential $\partial \widehat{\varphi_{m}}(\mathbf{x})$ to guide the unwrapping, the phase solution will be ambiguous at frequencies in the set $\left\{\boldsymbol{\omega} \in \mathbb{R}^{2}: \boldsymbol{\omega}=\pi \mathbf{M}, \forall \mathbf{M} \in \mathbb{Z}^{2}\right\}$, and it is not possible to determine the unknown coefficients without additional constraints.

The first and second phase differentials estimated from (3) and (4) and the spline derivative filters $H_{1}(z)$ and $H_{2}(z)$ developed in $[20,21]$ define two versions of the new phase unwrapping algorithm presented here. Although the second derivative version yields a phase solution that has fewer degrees of freedom, we have observed numerical inaccuracies in applying (4) to practical AM-FM components. Specifically, the second term in the equation is unstable at points with small AM values and where the modulating functions are not locally coherent. Moreover, the second term also contains the instantaneous bandwidth $\frac{\nabla a_{m}(\mathbf{x})}{a_{m}(\mathbf{x})}$, which was studied by Cohen [3] and is related to the range of values assumed by $\nabla \varphi_{m}(\mathbf{x})$ at a given point $\mathbf{x}$. Numerical excursions in the instantaneous bandwidth or the FM function $\nabla \varphi_{m}(\mathbf{x})$ will cause this second term to dominate the differential $\partial^{2} \widehat{\varphi_{m}}(\mathbf{x})$ and cause the least-squares unwrapping algorithm to yield a phase that does not agree with the structure of the image. We are currently studying the effects of constant-amplitude models for phase unwrapping because the instantaneous bandwidth is identically zero for this case; however, it is not yet clear under what conditions locally coherent AM-FM components can be adequately approximated by constant-amplitude models on small spatial neighborhoods. Thus, we employ the first derivative algorithm in the results presented here and note that we have obtained similar phase unwrapping results using the second derivative algorithm.

Regardless of which phase unwrapping algorithm is used, we enforce the congruency of the phase solution with the known principal values by embedding the principal values in the scaled phase solution $\gamma \psi_{m}(\mathbf{k})$ for some large $\gamma \in \mathbb{R}$. We define the phase modulation by

$$
\varphi_{m}(\mathbf{k})=W\left\{\varphi_{m}(\mathbf{k})\right\}+2 \pi b_{m}(\mathbf{k}) \approx \gamma \psi_{m}(\mathbf{k}),
$$


where $W\left\{\varphi_{m}(\mathbf{k})\right\}$ is the known wrapped phase and the branch function $b_{m}(\mathbf{k})$ is defined as

$$
b_{m}(\mathbf{k})=\operatorname{round}\left(\frac{\gamma \psi_{m}(\mathbf{k})-W\left\{\varphi_{m}(\mathbf{k})\right\}}{2 \pi}\right) .
$$

By taking the spline gradient of (7), we obtain the FM function $\nabla \varphi_{m}(\mathbf{x})$, which we can make arbitrarily close to the least-squares phase gradient by our choice of $\gamma$ :

$$
\left|\frac{1}{\gamma} \varphi_{m}(\mathbf{x})-\psi_{m}(\mathbf{x})\right| \leq \frac{\pi}{|\gamma|} .
$$

We choose $\gamma=300$ in practice, ensuring that the bound is generally less than $1 \%$, but independent of this value, the phase modulation $\varphi_{m}(\mathbf{k})$ can be recovered by integrating the spline gradient $\nabla \varphi_{m}(\mathbf{x})$. In this way, we can reconstruct each component $y_{m}(\mathbf{k})=a_{m}(\mathbf{k}) \exp \left[j \varphi_{m}(\mathbf{k})\right]$ without error.

\section{Results and Discussion}

Using the approach given in this paper, we have computed PR AM-FM models for a variety of images including the Lena and Cameraman images shown in Figs. 1(c) and 2(c). Computed modulating functions for the AM-FM image components shown in Figs. 1(d) and 2(d) are given in Figs. 1(e) and 2(e), respectively. The displays in Figs. 1(f) and 2(f) show the FM functions scaled by the corresponding AM functions, from which it is clear that the computed PR AM-FM models have succeeded in capturing the visually significant nonstationary structure of each image.

This is significant because it is the first demonstration of an invertible AM-FM image transform wherein the computed modulating functions fully characterize the visually important structure of the image. The next step in this work is to define perceptually meaningful image processing in terms of mathematical operations applied directly to the functions $a_{m}(\mathbf{x})$ and $\nabla \varphi_{m}(\mathbf{x})$ in the modulation domain.

\section{References}

[1] A. C. Bovik, N. Gopal, T. Emmoth, and A. Restrepo. Localized measurement of emergent image frequencies by Gabor wavelets. IEEE Trans. Info. Theory, 38(2):691-712, Mar. 1992.

[2] C. W. Chen and H. A. Zebker. Network approaches to twodimensional phase unwrapping: Intractability and two new algorithms. J. Opt. Soc. Am. A, 17(3):401-414, Mar. 2000.

[3] L. Cohen. Time-Frequency Analysis. Prentice Hall, Englewood Cliffs, NJ, 1995.

[4] D. Dimitriadis and P. Maragos. An improved energy demodulation algorithm using splines. In Proc. IEEE Int'l. Conf. Acoust., Speech, Signal Proc., pages 3481-3484, Salt Lake City, UT, May 7-11, 2001.
[5] T. J. Flynn. Two-dimensional phase unwrapping with minimum weighted discontinuity. J. Opt. Soc. Am. A, 14(10):2692-2701, Oct. 1997.

[6] D. C. Ghiglia and L. A. Romero. Robust two-dimensional weighted and unweighted phase unwrapping that uses fast transforms and iterative methods. J. Opt. Soc. Am. A, 11(1):107-117, Jan. 1994.

[7] J. P. Havlicek, D. S. Harding, and A. C. Bovik. Multidimensional quasi-eigenfunction approximations and multicomponent AM-FM models. IEEE Trans. Image Proc., 9(2):227242, Feb. 2000

[8] J. P. Havlicek, J. W. Havlicek, and A. C. Bovik. The analytic image. In Proc. IEEE Int'l. Conf. Image Proc., Santa Barbara, CA, Oct. 26-29, 1997.

[9] J. P. Havlicek, P. C. Tay, and A. C. Bovik. AM-FM image models: Fundamental techniques and emerging trends. In A. C. Bovik, editor, Handbook of Image and Video Processing, pages 377-395. Elsevier Academic Press, Burlington, MA, 2nd edition, 2005.

[10] S. Lu and P. C. Doerschuk. Nonlinear modeling and processing of speech based on sums of AM-FM formant models. IEEE Trans. Signal Proc., 44(4):773-782, Apr. 1996.

[11] P. Maragos and A. C. Bovik. Image demodulation using multidimensional energy separation. J. Opt. Soc. Amer. A, 12(9):1867-1876, Sep. 1995.

[12] P. Maragos, J. F. Kaiser, and T. F. Quatieri. Energy separation in signal modulations with applications to speech analysis. IEEE Trans. Signal Proc., 41(10):3024-3051, Oct. 1993.

[13] P. Maragos, J. F. Kaiser, and T. F. Quatieri. On amplitude and frequency demodulation using energy operators. IEEE Trans. Signal Proc., 41(4):1532-1550, Apr. 1993.

[14] R. A. Sivley and J. P. Havlicek. Multidimensional phase unwrapping for consistent APF estimation. In Proc. IEEE Int'l. Conf. Image Proc., pages 458-461, Genoa, Italy, Sep. 11-14, 2005, vol. II.

[15] S. M. H. Song, S. Napel, N. J. Pelc, and G. H. Glover. Phase unwrapping of MR phase images using Poisson equation. IEEE Trans. Image Proc., 4(5):667-676, May 1995.

[16] U. Spagnolini. 2-D phase unwrapping and phase aliasing. Geophys., 58(9):1324-1334, Sep. 1993.

[17] H. Takajo and T. Takahashi. Noniterative method for obtaining the exact solution for the normal equation in the leastsquares phase estimation from the phase difference. J. Opt. Soc. Am. A, 5(11):1818-1827, Nov. 1988.

[18] P. C. Tay and J. P. Havlicek. Joint uncertainty measures for maximally decimated M-channel prime factor cascaded wavelet filter banks. In Proc. IEEE Int'l. Conf. Image Proc., pages 1033-1036, Barcelona, Spain, Sep. 14-17, 2003, vol. I.

[19] P. C. Tay and J. P. Havlicek. Frequency implementation of discrete wavelet transforms. In Proc. IEEE Southwest Symp. Image Anal., Interp., pages 167-171, Lake Tahoe, NV, Mar. 28-30 2004

[20] M. Unser, A. Aldroubi, and M. Eden. B-spline signal processing: Part I-theory. IEEE Trans. Signal Proc., 41(2):821833, Feb. 1993.

[21] M. Unser, A. Aldroubi, and M. Eden. B-spline signal processing: Part II-efficient design and applications. IEEE Trans. Signal Proc., 41(2):834-848, Feb. 1993. 

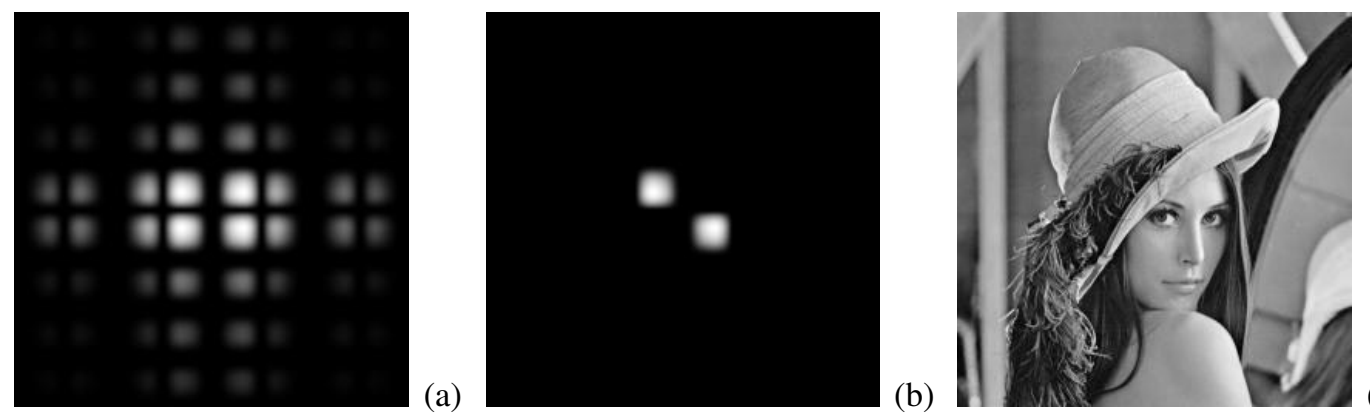

(c)

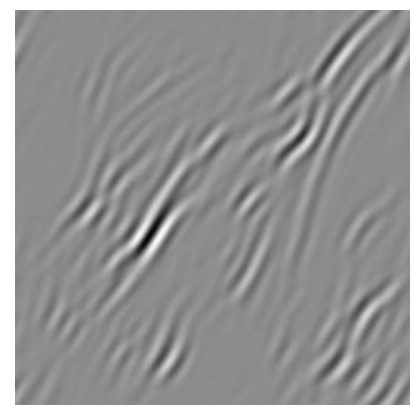

(d)
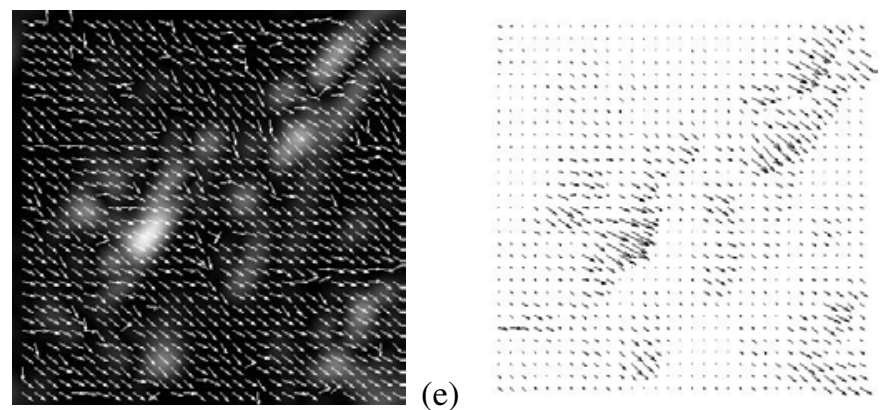

(f)

Figure 1. Lena example. (a) Frequency response of one of the separable wavelet filterbank channels. (b) One of the non-separable, orientation selective sub-channels obtained from (a). (c) Original Lena image. (d) AM-FM image component obtained from filtering image (c) with channel (b). (e) AM and FM functions computed from (d). (f) FM function scaled by the AM function.
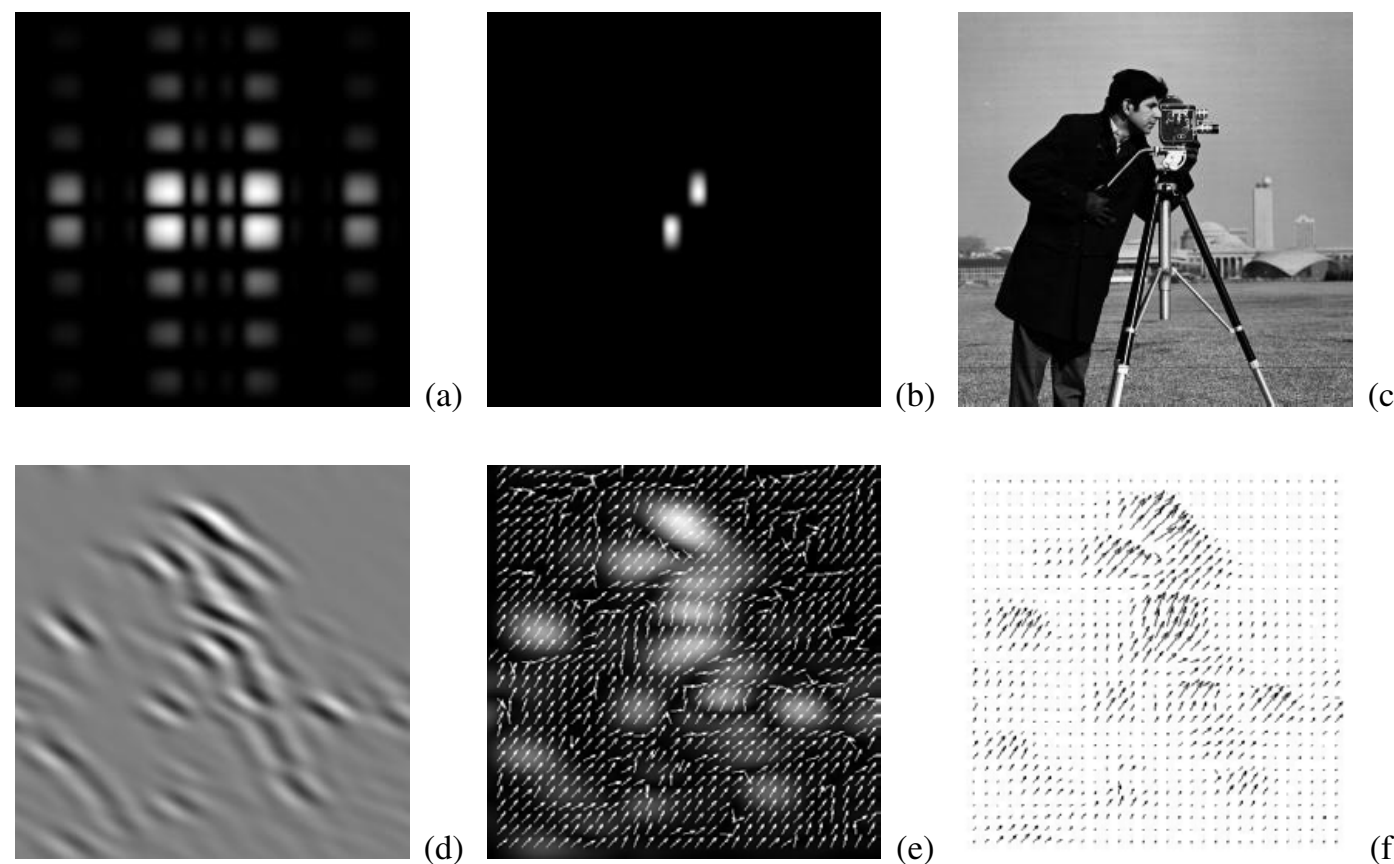

(c)
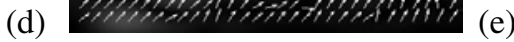

(f)

Figure 2. Cameraman example. (a) Frequency response of one of the separable wavelet filterbank channels. (b) One of the non-separable, orientation selective sub-channels obtained from (a). (c) Original cameraman image. (d) AM-FM image component obtained from filtering image (c) with channel (b). (e) AM and FM functions computed from (d). (f) FM function scaled by the AM function. 